Estudios Pedagógicos XXXIX, Número Especial 1: 103-119, 2013

INVESTIGACIONES

\title{
Competencias pedagógicas y función docente en las comunidades virtuales de aprendizaje
}

\author{
Pedagogical skills and teaching role in virtual learning communities \\ Competências pedagógicas eo papel de ensino em comunidades virtuais de aprendizagem
}

\author{
Siria Padilla P. , María Cristina López de la Madrid ${ }^{\mathrm{b}}$ \\ ${ }^{a}$ Centro Universitario de Ciencias Económico Administrativas (CUCEA). Universidad de Guadalajara. Telf.: 52 (33) 37703300. \\ Correo electrónico: siriapadilla@gmail.com \\ ${ }^{\mathrm{b}}$ Centro Universitario del Sur. Universidad de Guadalajara. Telf.: 52 (341) 57522 22. Correo electrónico: cristilm@ cusur.udg.mx
}

\section{RESUMEN}

El presente artículo es el resultado de una investigación que tuvo como punto de partida indagar sobre las competencias pedagógicas que deben desarrollar los profesores que trabajan con tecnologías de la información y, especialmente, nos referimos a las funciones de un profesor en una Comunidad Virtual de Aprendizaje (CVA). Se trata de un estudio de caso, con un enfoque cualitativo que analizó las estrategias discursivas utilizadas por el docente y estudiantes a lo largo de la actividad conjunta en un aula virtual, correspondiente a un curso regular de la Universitat Oberta de Catalunya (UOC).

Palabras clave: competencias pedagógicas, tecnologías de la información y la comunicación, función docente, comunidades virtuales de aprendizaje.

\begin{abstract}
This paper is the result of a research whose objective was to investigate the pedagogical skills to be developed by teachers working with information technology and, in particular, the functions of a teacher in a Virtual Learning Community (VLC). This is a case study with a qualitative approach which observed the discursive strategies used by the teacher and the students along the joint activity in a virtual classroom from a regular course of the Universitat Oberta of Catalunya (UOC).
\end{abstract}

Key words: pedagogical skills, information and communication technologies, teaching function, virtual learning communities.

\section{RESUMO}

Este artigo é o resultado de uma pesquisa que teve como ponto de partida aprofundar no conhecimento das competências pedagógicas desenvolvidas pelos professores que trabalham com tecnologias da informação com foco nas funções de um professor numa Comunidade Virtual de Aprendizagem (CVA). Este é um estudo de caso, com abordagem qualitativa que analisou as estratégias discursivas utilizadas pelo professor e alunos ao longo da atividade conjunta em uma sala de aula virtual correspondente a um curso regular da Universidade Aberta da Catalunha (UOC).

Palavras chave: competências pedagógicas, tecnologia da informação e comunicação, função de ensino, comunidades virtuais de aprendizagem. 


\section{INTRODUCCIÓN}

A partir del auge de internet, la Comunicación Mediada por Computadora ha ido ganando presencia como medio y apoyo a los procesos educativos que se realizan en los modelos mixtos, a distancia y presenciales enriquecidos. Con ella se hace referencia a las formas de comunicación asincrónica que mantienen una o más personas entre sí de forma escrita y que permiten a los sujetos formar redes electrónicas de aprendizaje, colaboración y participación. Gunawardena et al. (1997) lo definen como "el intercambio de mensajes entre un grupo de participantes por medio de redes electrónicas, con el propósito de discutir un tópico de mutuo interés" (p. 397). Por su parte Bustos et al. (2009) consideran que sus rasgos distintivos son: a) la no coincidencia espacio temporal entre sus participantes, b) la comunicación se basa en textos escritos, c) el intercambio de la información se realiza siempre a través de la computadora, d) permiten el almacenamiento de datos que se vuelven colectivos y que se ponen a disposición de todos para su constante revisión.

En ese marco, la formación de redes electrónicas entendida como la conexión entre dos o más ordenadores entre sí que comparten información, recursos y servicios, ha dado origen a una serie de usos y aplicaciones de las tecnologías; en este sentido Harasim et al. (1995) proponen al menos tres usos de las redes, estos son, en primer lugar, las aplicaciones que buscan compartir e intercambiar recursos entre instancias educativas para el apoyo a los modelos presenciales o a distancia. En segundo lugar, la creación de aulas o campus virtuales a través de los cuales se llevan a cabo los procesos de enseñanza aprendizaje y, en tercer lugar, la estructuración de redes de conocimiento (Knowledge Networks), identificadas como espacios a través de los cuales se puede producir la colaboración y la participación de los sujetos en red.

En este marco de crecimiento exponencial de las TIC, el profesorado cumple un papel relevante que lo hace actor principal en la inclusión e integración de las tecnologías, de ahí la importancia de identificar las funciones pedagógicas del profesor online, obteniendo con ello una caracterización y guía para el profesorado de esta área.

En este trabajo de investigación se analiza un aula virtual y, en específico, las funciones docentes que realiza un profesor en estos ambientes. Este acercamiento se realiza mediante un estudio de caso con enfoque cualitativo en el que se revisan las estrategias discursivas utilizadas por el profesor y los estudiantes en el desarrollo de la actividad conjunta del aula. El estudio de caso comprendió un aula virtual de la Universidad de Cataluña y las técnicas para recolectar datos correspondieron a la aplicación de cuestionarios a estudiantes y profesor, la observación participante, la recuperación y almacenamiento de documentos y/o mensajes relativos a los tres momentos del proceso de enseñanza aprendizaje: la planeación, el proceso de intervención y la evaluación. Para el análisis de datos, se utilizó como unidad de análisis el mensaje y se tomó como referente la propuesta de Coll y Onrubia (2001) sobre el uso de estrategias para la construcción del conocimiento compartido.

\section{COMUNIDADES VIRTUALES DE APRENDIZAJE}

Dentro de las aplicaciones tecnológicas que se han implementado en los últimos años, se encuentran las Comunidades Virtuales de Aprendizaje (CVA), caracterizadas por constituir más que una simple comunicación en red: son un conjunto o grupo de personas soportadas en la 
virtualidad y que mantienen relaciones entre sí, orientadas a compartir, generar y construir conocimiento a partir de las relaciones que establecen entre ellos y los intercambios comunicativos que direccionan (García, 2007). La importancia de las CVA es que han dado paso a la posibilidad de construcción de una subjetividad común a partir de que los sujetos conectados trabajen en una misma tarea o en un mismo fin, a pesar de la distancia y de los tiempos y ritmos diferenciados de trabajo.

Estos nuevos entornos virtuales pueden movilizar procesos intersubjetivos e intrasubjetivos que son fundamentales para la construcción social del conocimiento. Procesos intersubjetivos porque la dinámica de una red es la conexión entre las personas, el punto de encuentro entre sujetos distantes que, a través de puntos de concordancia, se reúnen y participan en procesos educativos comunes. Procesos intrasubjetivos porque la participación en esa construcción social del conocimiento ayuda a los sujetos a modificar sus propias estructuras cognitivas y a interiorizar el proceso social vivido, es decir, el proceso "exógeno" se vuelve "endógeno" al ser interiorizado en la conciencia de los sujetos. Para Coll (2010) una comunidad de aprendizaje:

...remite a la idea de un grupo de personas con diferentes niveles de experiencia, conocimiento y pericia que aprenden gracias a la colaboración que establecen entre sí, a la construcción del conocimiento colectivo que llevan a cabo y a los diversos tipos de ayuda que se prestan mutuamente (p. 7).

En éste sentido, para Garber (2004) una comunidad en línea o virtual no difiere significativamente de una comunidad física, sin embargo, su implementación y sus medios de interacción son diferentes, ya que se necesita compensar la falta de espacio físico y de comunicación sincrónica mediante la creación de nuevas estrategias de trabajo colaborativo.

Por su parte, Coll et al. (2008) señalan cuatro factores o características que ubican a las CVA en el interés actual:

1. La importancia otorgada al conocimiento y al aprendizaje.

2. La importancia de los factores contextuales, sociales, culturales, relacionales y colaborativos en los procesos de aprendizaje.

3. El acelerado desarrollo de las tecnologías de la información y comunicación.

4. La preocupación por la transformación y mejora de los sistemas educativos escolares y los centros de enseñanza.

De esta manera, y como eje central de estas CVA, el desarrollo tecnológico ha generado nuevos espacios de interacción y nuevas dinámicas de formación, transformando al aprendizaje en el medio de oportunidad para el desarrollo social, educativo y económico. De acuerdo con Barberà et al. (2001) las nuevas tecnologías han permitido plantearse el reto y la posibilidad de diseñar, planear y crear sistemas educativos virtuales desde una perspectiva constructivista, se trata de:

...espacios abiertos por las redes informáticas y diseñados sobe la base de tesis constructivistas para dar soporte a procesos didácticos, concebidos como dinámicas socioculturales, en que el proceso de enseñanza aprendizaje se considera inseparable de la situación en la que se produce, de la actividad que se desarrolla, de la interacción con las personas que intervienen en el proceso 
$y$ de la relación que se establece con los instrumentos culturales específicos incluidos en estos espacios (p. 81).

Para los autores citados, los contextos virtuales de enseñanza y aprendizaje constructivistas se caracterizan por:

a) Crear un marco de cooperación didáctica que debe entenderse como la interrelación entre los participantes para implicarse en las actividades educativas y de manera conjunta con el profesor. Se entiende que la cooperación didáctica es un proceso semiótico de negociación entre el aprendizaje y alumnos, mediado por el profesor y por las herramientas psicológicas las cuales deben ser las adecuadas para enriquecer y potenciar este proceso de negociación, partiendo del hecho de que cada sujeto tiene horizontes culturales definidos y saberes previos distintos.

b) Crear espacios de interacción que permitan a los alumnos trabajar en forma conjunta con el profesor y con los propios compañeros, es decir, aprovechar el espacio social del aula y sus encuentros para lograr que el estudiante se inserte en la actividad educativa. Se trata de que el alumno no trabaje de manera solitaria y autónoma, sino que el trabajo del aula le ayude a aprender.

c) Crear un equilibrio entre el material cultural que se pone a disposición del alumno para su propio aprendizaje y la guía y orientación del profesor. En la educación a distancia tradicional, la balanza se inclinaba por el trabajo más intensivo del alumno con el material. Actualmente se está buscando que exista una mayor orientación y guía del profesor, pero sin suprimir la importancia del propio control del alumno; en la educación mixta y presencial se busca, por el contrario, fortalecer el trabajo independiente del estudiante.

d) Asegurar que se desarrollen procesos de pensamiento complejos que impliquen una actividad de resolución en problemas en el análisis de casos, trabajos por proyectos "que tengan en cuenta la experiencia de los aprendices así como sus conocimientos adquiridos de manera informal que muchas veces prevalecen sobre los académicos" (p.83) y, que signifique para el profesor y el alumno una actividad intensiva en el desarrollo de estas habilidades.

e) Favorecer el desarrollo de una interacción orientada hacia la creación de zonas de desarrollo próximo. Se busca que el profesor otorgue ayudas ajustadas, entendidas como todo aquel soporte que confiere el profesor al estudiante y que varía de acuerdo al nivel de cada estudiante y/o del grupo en general, y a la evolución de la propia actividad desarrollada en el aula, se entiende que esas ayudas buscan lograr que el estudiante pase de un nivel de conocimiento a otro superior, pero, igualmente, implican formar comunidades virtuales de enseñanza y aprendizaje suficientemente ricas para lograr que las relaciones asimétricas entre estudiantes sean ocasión para la creación de zonas de desarrollo próximo entre pares.

f) Incluir tareas auténticas de aprendizaje que permitan diversificar los estilos de aprendizaje de los alumnos, evitar la excesiva textualidad así como el activismo inútil. Utilizar las tecnologías para lograr que los contenidos a aprender puedan estar orientados por su semejanza con la realidad.

Para Barberà et al. (2001) las CVA permiten la generación de contextos virtuales de aprendizaje que estarían constituidos por las interrelaciones posibles entre las características tecnológicas y pedagógicas, es decir, la relación entre las características propias de las herramientas tecnológicas y las posibilidades instruccionales que dan ocasión a dinámicas de enseñanza y aprendizaje que permiten el alcance de unos objetivos educativos específicos, tal como se muestra en la Figura 1. 
Figura 1. Esquema del contexto de aprendizaje con TIC

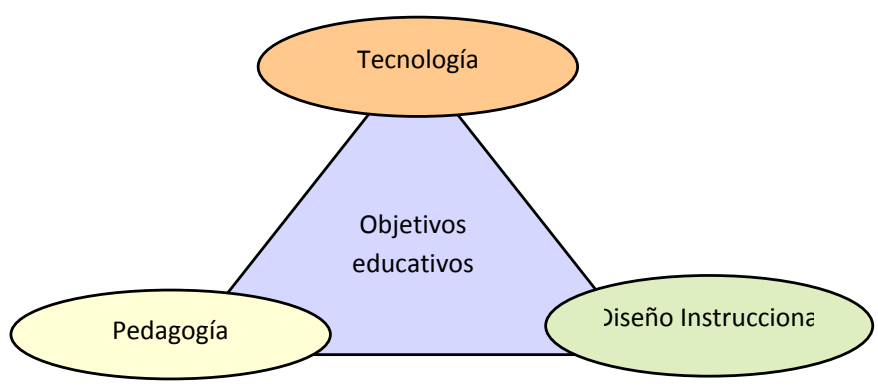

Fuente: Elaboración propia.

De hecho, la noción de contexto para los autores está ligada más que a las características tecnológicas del medio, al ambiente complejo y holístico en el que se produce la naturaleza del acto educativo, desde las condiciones más físicas, y por tanto objetivas, hasta las condiciones más subjetivas que se refieren al plano intersubjetivo de las actuaciones entre profesores y alumnos. Desde la perspectiva de los autores, en el contexto virtual de aprendizaje se incluyen además algunos factores como los recursos, la metodología y las formas de evaluación, así como las condiciones que se refieren a la forma en que se despliega la actividad pedagógica y que tiene que ver con las decisiones particulares que aparecen en el aula y que constituyen la diferencia entre el diseño y la actividad manifiesta en este espacio de aprendizaje.

En este sentido, Coll (2004) propone centrarse en los procesos de actuación conjunta que realizan profesor y alumnos en el seno de una actividad o tarea escolar, donde el medio tecnológico se incorpora como un instrumento o herramienta psicológica; por tanto, se centra más que en las características tecnológicas de la propia herramienta, en la forma en cómo es usada de manera conjunta por el profesor y el alumno en la consecución y logro de los objetivos de aprendizaje. Se estudia la forma en que se despliega esta actividad a través de y con apoyo en los medios tecnológicos, tomando en consideración que el medio tecnológico vehicula, permite y limita, de acuerdo a sus características, cierto tipo de actividades y aprendizajes, pero estos sólo se realizan y concretizan con apoyo en la actividad conjunta realizada por profesor y alumno.

De acuerdo con lo anterior y con las nuevas posibilidades constructivistas de las TIC, tendría que analizarse cuáles son esas competencias pedagógicas que deben fortalecer los profesores a fin de hacer real las potencialidades que las TIC proponen. A continuación, se analizan las competencias de los docentes en las comunidades virtuales de aprendizaje.

\section{COMPETENCIAS DOCENTES PARA EL TRABAJO EN LAS CVA}

Tanto en la educación presencial como en los entornos virtuales, las funciones del docente van más allá de la presentación de los contenidos y la comunicación casual con los alumnos. La complejidad de la sociedad, y por ende de los actuales sistemas educativos, ha generado demandas que hace 20 años no se habían hecho presentes, como lograr que los alumnos se interesen por aprender, pero que además puedan estructurar conceptos e ideas a partir de sus conocimientos previos; tener una interacción más dinámica y promover la participación activa de los educandos; comprender la importancia del acceso, selección y análisis de la información, así 
como lograr integrar los nuevos medios tecnológicos a las actividades educativas. Tal y como señalan Hannan y Silver (2006).

...el aprendizaje del estudiante ha dejado de ser el producto de un encuentro personal e individual cara a cara con el profesor, que ahora tiene lugar no solo en el auditorio para conferencias y en el laboratorio, sino también en grupos pequeños, en seminarios dirigidos por estudiantes, y en otras estructuras integradas en nuevos conceptos como el llamado "autoaprendizaje" (p. 84).

Así, y de acuerdo con Cabero 2007, "la movilización de estrategias es cada vez más importante ya que en un mundo repleto de información, y de una información que se transforma en cortos períodos de tiempo, más importante que el qué enseñar será cómo hacerlo y qué mecanismos utilizaremos para motivar y despertar el interés de los estudiantes" (p. 17).

En base a lo anterior y de acuerdo a lo que se señala desde la UNESCO (2005) en relación al uso de las TIC en la educación, el principal problema a abordar es contar con la experiencia adecuada por parte de los facilitadores a nivel individual, institucional y nacional, en términos de conocimientos, habilidades y actitudes porque, a decir de Cabero (2007) "al contrario de lo que cabría esperar con la aplicación de las TIC en la enseñanza, su utilización puede implicar la movilización de una diversidad de estrategias y metodologías docentes que favorezcan una enseñanza activa, participativa y constructiva" (p. 17).

Para Daura (2011), "así como es importante que el sujeto que aprende se esfuerce por ser estratégico, también lo es la estimulación que puede efectuar el agente de enseñanza por medio de las acciones que lleve a cabo" (p. 79). Si este requisito es necesario en la educación presencial, en las CVA resulta imprescindible, ya que, como hemos señalado, el docente debe de contar con una serie de habilidades y actitudes que lo lleven a trabajar con los alumnos de una forma más dinámica e integrada. De acuerdo con López y Chávez (2013), para potenciar el desarrollo del aprendizaje con el uso de las TIC, los docentes deben adquirir conocimientos en al menos 3 áreas:

- Conocimiento teórico pedagógico sobre las principales corrientes educativas, sus características, alcances y posibilidades.

- Conocimiento metodológico y didáctico para la integración adecuada de la tecnología a través de estrategias de enseñanza específicas.

- Conocimiento tecnológico básico, que no especializado, para identificar el alcance de las aplicaciones elementales y sus posibilidades de integración en los planes y programas de estudio.

Por su parte Cebrián (s.f.) señala que el docente debe de tener una preparación en al menos cinco dimensiones básicas:

1. Los procesos de comunicación y significación

2. Las formas y opciones de trabajar las TIC dentro de la disciplina escolar

3. La organización y didáctica sobre el uso de las TIC en el aula

4. Los conocimientos teórico-prácticos para analizar

5. Los criterios para la selección de materiales

En base a estas áreas de trabajo y formación expuestas por los autores, y desde un punto de vista holístico, la UNESCO (2005) define cuatro áreas de competencia en relación al docente y el uso de las TIC: 
1. Contenidos y Pedagogía centrados en las prácticas de enseñanza de los docentes y su conocimiento del plan de estudios. Se requiere que los profesores apliquen las TIC en sus respectivas disciplinas para apoyar y extender la enseñanza y el aprendizaje.

2. Colaboración y creación de redes para mostrar el potencial comunicativo de las TIC y hacerlo extensivo más allá del aula y las necesidades de desarrollo de nuevos conocimientos y habilidades.

3. Temas sociales que implican que los docentes puedan adquirir una comprensión de las cuestiones sociales, como los códigos legales y morales, los derechos de propiedad intelectual y derechos de autor, la participación en los debates sobre el uso y el impacto de las TIC, y el uso de las TIC en la promoción de una sociedad más sana. La conciencia de estos temas llevará a una aplicación adecuada de las TIC en la pedagogía y el desarrollo.

4. Cuestiones técnicas que incluyen la capacidad y la provisión tanto de la infraestructura como del soporte técnico para la integración de las TIC en el currículo (UNESCO, 2005, p. 12).

En este sentido, y así como los especialistas en el área de educación y TIC han formulado componentes, dimensiones y ejes concretos, a nivel gubernamental también se han realizado propuestas de integración sobre las necesidades de formación docente en TIC. Un ejemplo de ello lo observamos en el Centro de Educación y Tecnología de Chile, que presentó el documento Estándares en Tecnología de la Información y la Comunicación para la Formación Inicial Docente (MINEDUC, 2006), con 6 dimensiones de competencia docente:

1. Manejo y uso propiamente operativo de hardware y software, lo que en algunos casos viene articulado con la formación previa a la universidad;

2. Diseño de ambientes de aprendizaje, entendido como la habilidad y/o destreza para organizar entornos de enseñanza y aprendizaje con uso de tecnología;

3. Vinculación TIC con el currículum, donde se da importancia a realizar un proceso de aprendizaje desde las necesidades de los sectores curriculares (norma curricular) que permita contextualizar los aprendizajes;

4. Evaluación de recursos y aprendizaje centrado en las habilidades para evaluar técnica y críticamente el impacto del uso de ciertos recursos y organización de entornos de aprendizaje;

5. Mejoramiento profesional que refiere aquellas habilidades y destrezas que permiten a los docentes dar continuidad a lo largo de la vida a procesos de aprendizaje de /con TIC y,

6. Ética y valores orientados a contenidos legales y uso ético de recursos (p. 12).

Así, en relación a las actividades que los docentes realizan en las aulas virtuales, las competencias a desarrollar se deben de enfocar en la interacción con los alumnos, en la motivación para mantener el interés en los contenidos, y en las estrategias de enseñanza para lograr un mejor aprovechamiento. Khan (2005) propone 8 dimensiones ${ }^{1}$ involucradas en los procesos de aprendizaje mediado por TIC, una de las cuales es la pedagógica, que incluye aspectos analíticos de contenidos, de audiencia, de las metas de aprendizaje, de los medios y del diseño, así como estrategias instruccionales y organización. Este autor considera que el docente por sí mismo no puede atender todos los aspectos de esta dimensión, ya que es necesario que

\footnotetext{
${ }^{1}$ Las 8 dimensiones propuestas por Khan son: administración, evaluación, diseño de interface, tecnológica, institucional, ética, apoyo de recursos y pedagógica.
} 
dentro de un curso virtual, se desarrollen una serie de elementos de apoyo que confluyan en un aprendizaje más dinámico y auténtico.

En la parte que corresponde al docente, el autor se enfoca en la organización de los contenidos y en la variedad de las estrategias instruccionales que pueden ser desarrolladas para facilitar el aprendizaje y ayudar a los alumnos a lograr sus propias metas y objetivos educativos. Estos elementos son igualmente señalados por Coll et al. (2008), Coll (2008) y Barberà et al. (2001).

A partir de los anteriores elementos asociados con las necesidades de formación docente, y su relación con las propuestas de integración educativa de las TIC, Marquès (2000) señala de forma general algunas de las funciones pedagógicas que los docentes deben de llevar a cabo en su práctica, entre ellas:

- Diagnóstico de necesidades a partir del perfil de los estudiantes para conocer las características individuales y grupales; esto permite identificar las necesidades de formación del colectivo, lo que admite realizar una presentación adecuada de los contenidos y materiales del curso.

- Organización y gestión de los materiales, en donde las TIC tienen un rol central al permitir diversificar las opciones en las que se elaboran y distribuyen dichos materiales. En este sentido, los entornos virtuales son una opción para los programas que buscan descargar la presencialidad, y el docente debe contar con una preparación que le permita hacer un diseño adecuado de los contenidos de la asignatura.

- Motivación para despertar el interés de los estudiantes en los contenidos de la asignatura, e inducirlos a participar en acciones tendientes a enriquecerlos. El trabajo a través de las Comunidades Virtuales de Aprendizaje requiere de una atención continua hacia los estudiantes, por lo que se precisa de mayor apoyo para evitar la sensación de aislamiento y soledad.

- Orientación y tutorías mediante el seguimiento individual de los aprendizajes que proporcionen retroalimentación adecuada y oportuna. En este sentido, se busca incrementar el uso de las TIC para generar espacios de comunicación permanentes que permitan crear un clima de confianza y apertura.

- Desarrollo continuo, es decir, buscar nuevas estrategias y nuevas posibilidades para abordar los materiales y los contenidos, lo que implica integrarse en la realización de trabajos colaborativos con los estudiantes; realizar seguimientos a través de investigaciones para mejorar los procesos de enseñanza y aprendizaje; tener una capacitación continua; y fomentar actitudes positivas hacia las TIC.

Desde una perspectiva sociocultural, las funciones pedagógicas se relacionan con las ayudas ajustadas que otorga el profesor durante el proceso de enseñanza aprendizaje en el aula virtual, estas se entienden como ayudas que varían conforme se avanza en el propio desarrollo del grupo y/o del estudiante y, además, varían en su calidad pues se ajustan a las necesidades individuales o del grupo, por lo que se entiende que no se trata de capacidades genéricas sino de aplicaciones contextualizadas a las situaciones específicas de enseñanza y al proceso de aprendizaje de los estudiantes (Onrubia, 2000). La perspectiva constructivista de orientación sociocultural también entiende que todas las funciones pedagógicas otorgadas por el profesor están ligadas a la propia naturaleza de lo que se entiende por enseñanza y aprendizaje, así las ayudas otorgadas por un profesor de orientación constructivista diferirán de aquel que considera que la enseñanza consiste en una transmisión de contenidos. 
De acuerdo con lo anterior, hace falta analizar cómo se pueden utilizar las TIC en una CVA para que apoyen la generación y construcción del conocimiento genuino del estudiante. En el siguiente apartado, se realizan algunas aportaciones a este respecto a partir del análisis de datos empíricos arrojados por la presente investigación.

\section{RESULTADOS GENERALES}

La importancia de las TIC en educación se encuentra en su capacidad para crear comunicación asincrónica que apoya la creación de procesos de intersubjetividad entre los miembros de las CVA, es decir, la posibilidad de crear comunicación didáctica entre profesor y estudiantes que redunde en una mejora de los procesos de enseñanza aprendizaje. De ahí la posibilidad que ofrecen las TIC para crear CVA de tipo constructivista, en el que los procesos comunicativos, el enriquecimiento del material didáctico y las oportunidades de diseños auténticos generan nuevos ambientes enriquecidos.

En este apartado, exponemos algunos hallazgos de investigación en torno a la función docente que realiza el profesor en una CVA, dicha investigación se centró en el estudio de los mecanismos que utilizaba el profesor para apoyar el proceso de enseñanza aprendizaje en un aula virtual y pueden agruparse en las siguientes dimensiones: a) acciones para organizar y gestionar la actividad en el aula virtual, b) acciones para orientar y dar sentido al aprendizaje, c) acciones de apoyo al clima socioemocional del aula, d) acciones para la exploración de los conocimientos previos de los estudiantes, e) acciones al servicio de la elaboración de representaciones cada vez más complejas y expertas de contenidos de enseñanza aprendizaje. Dichas dimensiones se obtuvieron a partir de la propuesta de Coll y Onrubia (2001), no obstante se ajustaron y modificaron a partir de la propia evidencia empírica encontrada. El análisis de las ayudas se centró en las estrategias discursivas utilizadas por los docentes para guiar y conducir el proceso de enseñanza aprendizaje en las CVA; a continuación explicamos cada uno de ellos.

\subsection{ORGANIZACIÓN Y GESTIÓN DE LAS ACTIVIDADES EN LAS AULAS VIRTUALES}

Garrison y Anderson (2005) reconocen que uno de los roles importantes del profesor está relacionado con la organización y gestión de la actividad del aula, la cual incorporan en la categoría de "presencia docente". Las funciones docentes que incluyen en esta dimensión son el diseño y la organización: "el diseño se refiere a las decisiones estructurales adoptadas antes de que comience el proceso, mientras que la organización se refiere a decisiones similares que son tomadas para adaptarse a los cambios durante la transacción educativa” (p. 99).

De acuerdo con nuestros hallazgos, se trata de un tipo de actividad que tiene por objeto poner las bases para el trabajo conjunto, generar las condiciones de qué se hace y cómo se hace, marcar el inicio de una actividad o el cambio de actividades. A esta actividad áulica corresponde una estructura discursiva que Cross (2002) denomina "estructura directiva o instruccional", la cual sirve para organizar la actividad en clase y contiene una secuencia de órdenes que indican el lugar, tiempo y manera de realizar un ejercicio o actividad, así como su justificación argumentativa. En las aulas virtuales el profesor utiliza estrategias discursivas directivas o instruccionales para organizar la gestión de la clase con el fin de señalar órdenes e instrucciones en la realización de actividades y ejercicios (ver Tabla 1). 
La organización y gestión de las actividades, como lo menciona Castellà (2007), comprende todas aquellas explicaciones y orientaciones del profesor que establecen las bases para el trabajo conjunto, generar sus condiciones y normas, así como su apertura, continuidad y buen funcionamiento. Se busca establecer qué se debe hacer, con qué recursos, materiales, espacios, sujetos; la organización del aula virtual tiene que ver con los materiales, las guías y los recursos, su organización y disposición espacial.

Tabla 1. Estrategias de organización y gestión de la actividad

\begin{tabular}{|l|l|}
\hline Descripción & Funciones \\
\hline $\begin{array}{l}\text { Instrucciones dirigidas a los estudiantes } \\
\text { sobre qué hacer, cuándo y en qué espacio. }\end{array}$ & Organización de la actividad en el aula. \\
\hline $\begin{array}{l}\text { Instrucciones dirigidas a los estudiantes } \\
\text { sobre las reglas de participación en el aula. }\end{array}$ & $\begin{array}{l}\text { Organizar el trabajo colectivo en los foros } \\
\text { de discusión y/o en pequeños grupos. }\end{array}$ \\
\hline $\begin{array}{l}\text { Aclaración sobre las actividades: tiempos, } \\
\text { características del trabajo y vías de entrega. }\end{array}$ & $\begin{array}{l}\text { Aclarar los tiempos, espacios y } \\
\text { características del trabajo. }\end{array}$ \\
\hline $\begin{array}{l}\text { Llamadas de atención a los estudiantes por } \\
\text { acciones incorrectas dentro del marco de las } \\
\text { reglas establecidas. }\end{array}$ & $\begin{array}{l}\text { Amonestar a los estudiantes por realizar } \\
\text { acciones incorrectas. }\end{array}$ \\
\hline $\begin{array}{l}\text { Negociación con los estudiantes sobre los } \\
\text { periodos y/o espacios de entrega de } \\
\text { actividades. }\end{array}$ & $\begin{array}{l}\text { Permitir flexibilidad al estudiante sobre su } \\
\text { propia actividad. }\end{array}$ \\
\hline
\end{tabular}

Fuente. Elaboración propia.

Como podemos observar, el trabajo simultáneo y el uso de foros para constituir la actividad multiespacial requieren de una organización para su uso óptimo, qué materiales se deben usar y para qué actividad, dónde se encuentran localizados, cuáles son los recursos para trabajar una actividad. Por otro lado, qué tipo de discurso se debe utilizar, con qué rigor, cómo debe estar organizado y cuántas veces se debe participar, son algunas de las estrategias que organizan la actividad conjunta en las aulas virtuales. Se incluyen también aquí el diálogo entre profesor y estudiantes en torno a la comprensión de la actividad, Barberà et al. (2001) denominan a estas interacciones como "el proceso comunicativo que se establece ente varias personas dentro de un contexto virtual cuando tienen por finalidad la progresiva definición de las condiciones que enmarcan la situación dentro de la cual se ha de realizar la tarea" (p. 182).

\subsection{ORIENTAR Y DAR SENTIDO AL APRENDIZAJE}

Una parte importante de la construcción del conocimiento sucede cuando los estudiantes otorgan un sentido positivo a su aprendizaje, éste no ocurre de manera natural en el aula, pero es fundamental para abordar la actividad con un enfoque pertinente. De ahí que la implicación del profesor sea importante para orientar las actividades, explicarlas y señalar su relevancia para la adquisición de los contenidos del curso, para su desarrollo profesional y para el logro de habilidades y destrezas. En este sentido, y como señala Coll y Onrubia (2001): 
Que el alumno atribuya un sentido positivo a su aprendizaje, que adopte una disposición favorable a implicarse en el proceso de aprender y que aborde el proceso de aprendizaje con la intención de comprender en profundidad el contenido de que se trate no es sencillo ni está en absoluto garantizado de antemano (p. 27).

En las aulas virtuales, la separación física entre profesor y estudiantes hace que estos últimos tengan que tener contacto directo primero con las guías y planes y, a través de éstas, formarse una representación de la actividad o la tarea a realizar; sin embargo, se requiere que el alumno asuma un enfoque profundo en la realización de la misma y esto se logra a través del apoyo del profesor. De esta manera, las acciones del profesor están dirigidas a otorgar sentido a la actividad inmediata, a orientar la actividad para que los alumnos la consideren relevante en su desarrollo o aprendizaje, es decir, que asuman un enfoque profundo y caractericen el conocimiento como compartido, esto último consiste, de acuerdo con Mercer (1995), en

ayudar a los alumnos a ver que las distintas actividades que hacen en el tiempo contribuyen al desarrollo de su comprensión. La educación no puede ser meramente la experiencia de una serie de hechos consecutivos, debe ser un proceso de desarrollo en el que las experiencias anteriores proporcionan las bases para dar sentido a las siguientes (p. 44).

Si bien Mercer se refiere a las aulas presenciales, en los hallazgos detectados de la investigación se encontró que también se utilizan estas técnicas por parte del profesor y de los estudiantes para conducir el proceso de enseñanza (ver Tabla 2).

Tabla 2. Estrategias para orientar y dar sentido a las actividades

\begin{tabular}{|l|l|}
\hline Descripción & Funciones \\
\hline $\begin{array}{l}\text { Meta-enunciados a partir de los cuales se } \\
\text { explican las actividades que realizarán los } \\
\text { estudiantes en el marco de un módulo, una } \\
\text { unidad, o un curso. }\end{array}$ & $\begin{array}{l}\text { Dar sentido a la actividad del estudiante, } \\
\text { establecer una visión general del curso y/o } \\
\text { del módulo. }\end{array}$ \\
\hline $\begin{array}{l}\text { Explicar la importancia de una actividad o } \\
\text { tarea en relación a los aprendizajes } \\
\text { esperados y/o al perfil profesional esperado. }\end{array}$ & $\begin{array}{l}\text { Lograr que los estudiantes asuman un } \\
\text { enfoque profundo de aprendizaje, logrando } \\
\text { ir más allá de las características formales de } \\
\text { la tarea. }\end{array}$ \\
\hline $\begin{array}{l}\text { Otorgar sentido a la actividad inmediata a } \\
\text { partir de su relación con la actividad } \\
\text { conjunta anterior. }\end{array}$ & $\begin{array}{l}\text { Caracterizar la actividad como una acción } \\
\text { conjunta y progresiva. }\end{array}$ \\
\hline
\end{tabular}

Fuente: Elaboración propia.

Otorgar sentido al aprendizaje constituye una vía destacada para que los estudiantes se impliquen en la actividad, máxime en una CVA donde existe mayor peligro de carecer de una perspectiva de esta naturaleza. 


\subsection{APOYO AL CLIMA SOCIOEMOCIONAL DEL AULA}

Este conjunto de estrategias están relacionadas a la parte emocional y afectiva con las que se abordan las tareas o actividades escolares, y con la necesidad de dar cohesión a un grupo. Gunawardena (1995) denomina presencia social a la habilidad de hacerse presente o real en una comunicación mediada por computadora. Abatir la distancia y la separación física y psicológica entre el profesor y el estudiante supone poner en marcha una serie de estrategias que permitan la comunicación "emocional" entre los actores. Garrison y Anderson (2005) siguiendo a Gunawardena utilizan también el término presencia social y lo asocian con categorías como afecto, comunicación abierta y cohesión.

Para las autoras de este artículo, algunas de las estrategias utilizadas para apoyar el clima socioemocional del aula son el re-conocimiento de la identidad de sus miembros, es decir, poder identificar quiénes son los sujetos que participan en el aula, lo cual resulta muy importante en las aulas virtuales, dada la separación física entre los sujetos; en segundo lugar, el soporte emocional que consiste en otorgar apoyos a los sujetos que, por diversas circunstancias, se encuentran en situaciones de estrés, de conflicto, o de malestar emocional, ya sea resultado de sus condiciones personales y/o de condiciones institucionales y académicas; en tercer lugar, gestionar y crear un vínculo entre los participantes que permita la cohesión del grupo, y el establecimiento de condiciones favorables para la comunicación y la colaboración entre los participantes del acto educativo (ver Tabla 3).

Tabla 3. Estrategias de apoyo al clima socioemocional del aula

\begin{tabular}{|l|l|}
\hline Descripción & Funciones \\
\hline $\begin{array}{l}\text { Presentación de estudiantes y del profesor } \\
\text { en los foros. }\end{array}$ & $\begin{array}{l}\text { Identificarse en el aula virtual, saber } \\
\text { quiénes son los integrantes de la comunidad } \\
\text { educativa. }\end{array}$ \\
\hline $\begin{array}{l}\text { Otorgar ayudas a los estudiantes en caso de } \\
\text { estrés, angustia, frustración, etc. }\end{array}$ & $\begin{array}{l}\text { Otorgar soporte emocional al estudiante, dar } \\
\text { continuidad a la actividad educativa. }\end{array}$ \\
\hline $\begin{array}{l}\text { Intercambiar ficheros: música, frases, } \\
\text { vídeos. }\end{array}$ & $\begin{array}{l}\text { Apoyar la integración del grupo y su } \\
\text { cohesión. }\end{array}$ \\
\hline $\begin{array}{l}\text { Solicitar la colaboración de todos, animar a } \\
\text { ayudarse mutuamente y a colaborar entre sí. }\end{array}$ & $\begin{array}{l}\text { Apoyar la cohesión del grupo a través del } \\
\text { apoyo mutuo. }\end{array}$ \\
\hline
\end{tabular}

Fuente: Elaboración propia.

A través de estas estrategias se destaca que el aprendizaje no sólo es un acto cognitivo sino también socioemocional, por lo que habría que poner mayor énfasis en éste como apoyo a los procesos de construcción del conocimiento.

\subsection{EXPLORACIÓN Y ACTIVACIÓN DE LOS CONOCIMIENTOS PREVIOS DE LOS ALUMNOS}

La visión constructivista del conocimiento parte del reconocimiento de que el aprendizaje es un proceso por el cual los sujetos elaboran representaciones personales sobre la realidad o 
sobre el contenido o tarea que se pretende conocer (Solé y Coll, 2000). Por su parte, el acto de conocer es una construcción situada y social (Cubero et al., 2007) por la cual las personas crean su propia experiencia con base en sus propios conocimientos, habilidades y actitudes. A su vez, esta construcción personal se realiza a partir de las interacciones de carácter social que se establece con los otros. En el aprendizaje como proceso constructivo juegan un papel destacado las propias representaciones o conocimientos que el estudiante posee, pues como señala Coll (citado por Miras, 2000):

Cuando el alumno se enfrenta a un nuevo contenido a aprender, lo hace siempre armado con una serie de conceptos, concepciones, representaciones y conocimientos adquiridos en el transcurso de sus experiencias previas, que utiliza como instrumentos de lectura e interpretación y que determinan en buena parte qué informaciones seleccionará, cómo las organizará y qué tipos de relaciones establecerá entre ellas (p. 50).

Estos conocimientos previos son los que le permiten hacer los puentes entre sus propios saberes y los contenidos a aprender, por ello es necesario que el profesor y los estudiantes, de manera conjunta, movilicen esos saberes previos con la finalidad de que los nuevos contenidos puedan adquirir significado. Un aprendizaje es significativo solo cuando los nuevos conocimientos se vinculan con los saberes previos y pre-existentes.

La investigación ha revelado que profesores y estudiantes construyen niveles de intersubjetividad inicial que les permiten avanzar en el conocimiento, esa creación de marcos de referencia comunes puede ser general o específica; son generales cuando atañen a conocimientos o saberes externos, propios de la cultura, y son específicos cuando se han construido a lo largo de la propia experiencia áulica virtual (Mercer, 1995).

Tabla 4. Estrategias de exploración y activación de conocimientos previos

\begin{tabular}{|l|llll|}
\hline Descripción & Funciones & & & \\
\hline $\begin{array}{l}\text { Los profesores y/o los alumnos aluden al } \\
\text { contexto sociocultural: } \\
\text { experiencias como pre-supuesto cultural } \\
\text { compartido. }\end{array}$ & $\begin{array}{l}\text { Construcción de un marco general de } \\
\text { referencia común. }\end{array}$ & & \\
\hline $\begin{array}{l}\text { Los profesores y los alumnos aluden a } \\
\text { conocimientos, acciones y experiencias que } \\
\text { fueron objeto de una experiencia común } \\
\text { compartida en el aula. }\end{array}$ & $\begin{array}{l}\text { Construcción de marco específico de } \\
\text { referencia común. }\end{array}$ & & \\
\hline $\begin{array}{l}\text { El profesor y/o los estudiantes solicitan } \\
\text { información necesaria sobre la experiencia } \\
\text { y/o conocimientos previos de los }\end{array}$ & & & & \\
estudiantes.
\end{tabular}

Fuente: Elaboración propia.

Como podemos observar en la Tabla 4, los estudiantes en estos espacios comunican sus propias representaciones sobre las tareas, actividades o conocimientos y buscan compartir y confirmar sus propias representaciones con el profesor y con el resto del grupo. Esas 
representaciones iniciales son importantes pues permiten establecer niveles de intersubjetividad común que permita asegurar a los miembros de la comunidad de qué se habla y cómo se entiende de lo que se está hablando. Por otra parte, el profesor obtiene información relevante de sus estudiantes, para ello realiza preguntas que buscan asegurarse sobre qué saben los estudiantes, qué tanto saben de ello, y cómo entienden dichos contenidos.

\subsection{APOYO EN LA PROFUNDIZACIÓN Y ELABORACIÓN DE REPRESENTACIONES MÁS COMPLEJAS Y EXPERTAS DEL CONTENIDO DE ENSEÑANZA Y APRENDIZAJE}

Una vez que los estudiantes han avanzado en la creación de un nivel inicial de intersubjetividad, los alumnos avanzan hacia niveles más complejos de representación del conocimiento. Ese proceso no se produce de manera espontánea y es el profesor junto a los estudiantes quienes ponen en funcionamiento diversas estrategias discursivas que les permiten dar este paso. Si bien los conocimientos previos son importantes para la activación del aprendizaje significativo, también se requieren diversas estrategias discursivas para lograr pasar a niveles de complejidad crecientes en la representación del conocimiento; ¿cuáles son esas estrategias, quién las moviliza, cómo se producen?, son algunas de las preguntas que podemos hacernos sobre esta cuestión, también es importante saber por qué no siempre se llega a estos niveles de complejización y qué los favorece.

En las representaciones iniciales, los estudiantes recurren a su propia experiencia, a su conocimiento cotidiano, sin embargo en las formas discursivas más complejas se busca que el estudiante se aleje de esas representaciones cotidianas más próximas y se acerque a un conocimiento más científico. Las estrategias discursivas que utilizan profesores y alumnos para estos propósitos son: a) ampliación del horizonte de comprensión de los estudiantes, b) legitimación del conocimiento científico, c) realización de recapitulaciones y síntesis, d) desarrollo argumentativo del punto de vista de los estudiantes y/o del profesor, que a continuación detallamos y que se muestran en la tabla 5:

Tabla 5. Estrategias de apoyo en la profundización y elaboración de representaciones más complejas y expertas del contenido de enseñanza aprendizaje

\begin{tabular}{|l|l|}
\hline Descripción & Funciones \\
\hline $\begin{array}{l}\text { Se detectan incomprensiones por parte del } \\
\text { profesor y/o los estudiantes respecto a un } \\
\text { contenido o una actividad. }\end{array}$ & $\begin{array}{l}\text { Ampliar el horizonte de comprensión del } \\
\text { estudiante. }\end{array}$ \\
\hline $\begin{array}{l}\text { Se formulan preguntas por parte del } \\
\text { profesor y/o los estudiantes con el objeto de } \\
\text { solicitar explicaciones argumentadas de sus } \\
\text { puntos de vista. }\end{array}$ & $\begin{array}{l}\text { Se busca propiciar la reflexión y el debate } \\
\text { argumentado. }\end{array}$ \\
\hline $\begin{array}{l}\text { El profesor utiliza apoyos en fuentes } \\
\text { científicas, etiqueta palabras vertidas por los } \\
\text { estudiantes que lo orienten a un enfoque } \\
\text { científico. }\end{array}$ & $\begin{array}{l}\text { Se orienta al estudiante a una construcción } \\
\text { cientica del conocimiento. }\end{array}$ \\
\hline Se realizan reformulaciones de las & Se valida el conocimiento vertido por los \\
\hline
\end{tabular}




\begin{tabular}{|l|l|}
\hline aportaciones de los estudiantes. & estudiantes. \\
\hline $\begin{array}{l}\text { El profesor realiza síntesis o } \\
\text { recapitulaciones del trabajo realizado en el } \\
\text { aula. }\end{array}$ & $\begin{array}{l}\text { Se busca hacer énfasis en las ideas } \\
\text { relevantes aportadas por los estudiantes y/o } \\
\text { profesor respecto a los contenidos y/o } \\
\text { actividades. }\end{array}$ \\
\hline
\end{tabular}

Fuente: Elaboración propia.

En este sentido, el estudiante va tomando un papel cada vez más activo en el que sus destrezas y habilidades se deberán reflejar en el sentido que vaya dando a la información recibida, tanto desde la cotidianeidad como desde la ciencia. La construcción de nuevos conceptos se irá facilitando en la medida en que éste pueda interiorizar los caminos para el análisis, síntesis y apropiación de los contenidos recibidos.

\section{CONCLUSIONES}

Las TIC han creado y posibilitado la creación de ambientes virtuales de aprendizaje con enfoques constructivistas en los que se resalta la utilización de materiales educativos enriquecidos, la posibilidad de crear intersubjetividad entre profesor y estudiantes a partir de la creación de diálogo común didáctico, y la creación de diseños de aprendizaje que emulen la complejidad del mundo real. En todo ello, el profesor debe desarrollar ciertas competencias pedagógicas que se consideran ejes motores del saber y del hacer docente.

En el campo de la virtualidad, y en específico de las CVA, hace falta identificar qué es lo que debe realizar un profesor, pues el alto énfasis dado a la tecnología y a sus atributos hizo que éste pasara a un segundo plano. En este sentido, la actitud del docente es un elemento central para el buen o mal aprovechamiento de la tecnología en el aula, pues aquellos docentes, que en palabras de Umberto Eco son los integrados, manifestarán una disposición abierta hacia nuevas posibilidades de trabajo con TIC; en contraparte, si el docente no está a favor de esta integración tecnológica, hará lo posible por obstaculizar su uso en el aula. De esta manera, UNESCO (2011) ha señalado que más allá de las competencias técnicas y de acompañamiento, la utilización eficaz de las tecnologías en el aula depende, en última instancia, de la motivación de los docentes.

En relación a lo anterior, el enfoque constructivista resalta el papel del docente en la activación del conocimiento del estudiante, en la capacidad de éste para crear un ambiente propicio, en la activación y profundización del conocimiento, pero como establece Vaello (2011), ese desarrollo cognitivo sólo es posible cuando también se ha insistido en la atención socioemocional del grupo y en la gestión y organización de la clase, aspectos que en conjunto permiten la construcción del conocimiento en las aulas. Si como señalan Coll et al. (2008), una CVA es un espacio de oportunidad para colaborar con otros y aprender de otros, así como para construir soluciones innovadoras a los problemas sociales y educativos, los docentes tienen ante sí una ocasión favorable para incidir en la formación de sus estudiantes de una forma más abierta, corresponsable y dinámica. 


\section{REFERENCIAS BIBLIOGRÁFICAS}

Barberà, E. (Coord.), Badia, A. y Mominó, J. (2001). La incógnita de la educación a distancia. Barcelona: ICE-Horsori, Universitat de Barcelona.

Bustos, A., Coll, C. y Engel, A. (2009). Presencia docente distribuida en redes asincrónicas de aprendizaje. Definición teórica y perspectiva multi-método para su estudio. En F. Díaz Barriga., G. Hernández. y M. Rigo (Eds.), Aprender y enseñar con TIC en educación superior: Contribuciones al socio-constructivismo (pp. 97-148). Ciudad de México: Universidad Nacional Autónoma de México, Facultad de Psicología, DGAPA.

Cabero, J. (Coord.). (2007). Nuevas Tecnologías Aplicadas a la Educación. España: Mc Graw Hill.

Castellà, J., Comelles, S., Cros, A. y Vilá, M. (2007). Entender(se) en clase. Las estrategias comunicativas de los docentes bien valorados. Barcelona: Graó.

Cebrián de la Serna, M. (s.f.). Una nueva necesidad, una nueva asignatura. Málaga: Universidad de Málaga. Recuperado de http://www.uib.es/depart/gte/cebrian.html.

Coll, C. (2010). Enseñar y aprender en el mundo actual: desafíos y encrucijadas. Pensamiento Iberoamericano, $n$. 7, 47-66.

. (2004). Las comunidades de aprendizaje. Nuevos horizontes para la investigación y la intervención en psicología de la educación. IV Congreso Internacional de Psicología y Educación. Simposio: Nuevos horizontes en Psicología de la Educación. Almería, 30-31 de marzo y 1-2 de abril de 2004. Recuperado de: http://www.ub.edu/grintie.

Coll, C., Bustos, A. y Engel, A. (2008). Las comunidades virtuales de aprendizaje. En C. Coll y C. Monereo (Eds.), Psicología de la educación virtual (pp. 299-320). Morata: Madrid.

Coll, C., Onrubia, J. (2001). Estrategias discursivas y recursos semióticos en la construcción de sistemas de significados compartidos entre profesor y alumnos. Revista investigación en la escuela, $n$. 45, 21-31.

Cros, A. (2002). La clase magistral. En C. Lomas (Ed.), El aprendizaje de la comunicación en las aulas (pp. 209-224). Barcelona: Paidós.

Cubero R., Cubero, M., Santamaría, A., Saavedra, J. y Bernal, J. (2007). Aprendizaje y psicología histórica-cultural. Aportaciones de una perspectiva social del aula. Investigación en la Escuela, n.62, 5-16.

Daura, F. (2011). Las estrategias docentes al servicio del desarrollo del aprendizaje autorregulado. Estudios pedagógicos, vol.XXXVII, n.2, 77-88.

Eco, Umberto (1995). idos. España: Tusquets Editores.

Garber, D. (2004). Growing virtual communities. International Review of Research in Open and Distance Learning, vol.5, n2. Recuperado de http://www.irrodl.org/index.php/irrodl/article/view/177/259

García, A. (Ed.), Ruiz, C. y Domínguez, F. (2007). De la educación a distancia a la educación virtual. Barcelona: Ariel.

Garrison, D. y Anderson, T. (2005). El e-learning en el siglo XXI. Investigación y práctica. Barcelona: Octaedro. 
Gunawardena, C. (1995). Nuevos caminos en el aprendizaje, nuevas formas de evaluar. En P.J. Dirr y C.N. Gunawardena (Eds.), Cuadernos de educación a distancia 3: Enfoques sobre evaluación de los aprendizajes en educación a distancia II (pp. 57-83). México: Universidad de Guadalajara - CECAD.

Gunawardena, C., Lowe, C. y Anderson, T. (1997). Analysis of a global online debate and the development of an interaction analysis model for examining social construction of knowledge in computer conferencing. Journal Educational Computing Research, vol. 17, n. 4, 397-431.

Hannan, A. y Silver, H. (2006). La innovación en la Enseñanza Superior. Enseñanza, aprendizaje y culturas institucionales. España: Narcea Ediciones.

Khan, Badrul (2005). Managing E-learning Strategies. U.S.A.: Information Science Publishing.

López, C. y Chávez, J. (2013). La formación de profesores universitarios en la aplicación de las TIC. Sinéctica, $\quad 4 . \quad$ Recuperado de http://www.sinectica.iteso.mx/?seccion=articulo\&lang=es\&id=609_la_formacion_de_pro fesores_universitarios_en_la_aplicacion_de_las_tic

Marquès, P. (2000). Los docentes: funciones, roles, competencias necesarias, formación. Documento de trabajo. Universidad Autónoma de Barcelona. Recuperado de: http://peremarques.pangea.org/docentes.htm.

Mercer, N. (1995). La construcción guiada del conocimiento. El habla de profesores y alumnos. Barcelona: Paidós.

Ministerio de Educación de Chile. (2006). Estándares en Tecnología de la Información y la Comunicación para la Formación Inicial Docente. Santiago de Chile: Ministerio de Educación. $\quad$ Recuperado de http://www.enlaces.cl/portales/tp3197633a5s46/documentos/200707191420080.Estandares.p df.

Miras, M. (2000). Un punto de partida para el aprendizaje de nuevos contenidos: Los conocimientos previos. En C. Coll, E. Martín, T. Mauri, M. Miras, J. Onrubia, I. Solé y A. Zabala, El constructivismo en el aula (pp. 47-64). Barcelona: Graó.

Onrubia, J. (2000). Enseñar: crear zonas de desarrollo e intervenir en ellas. En C. Coll, E. Martín, T. Mauri, M. Miras, J. Onrubia, I. Solé y A. Zabala, El constructivismo en el aula (pp. 101-124). Barcelona: Graó.

Solé, I. y Coll, C. (2000). Los profesores y la concepción constructivista. En C. Coll, E. Martín, T. Mauri, M. Miras, J. Onrubia, I. Solé y A. Zabala, El constructivismo en el aula (pp. 723). Barcelona: Graó.

UNESCO. (2005). Regional Guidelines on Teacher Development for Pedagogy-Technology Integration (Working Draft). Thailand: Autor.

UNESCO. (2011). Transforming Education: The Power of ICT Policies. France: Autor.

Vaello, O. (2011). Cómo dar clase a los que no quieren. España: Graó. 